\title{
Effect of Intensive and Standard Pitavastatin Treatment With or Without Eicosapentaenoic Acid on Progression of Coronary Artery Calcification Over 12 Months
}

\author{
- Prospective Multicenter Study -
}

\author{
Toru Miyoshi, MD; Kunihisa Kohno, MD; Hirohiko Asonuma, MD; Satoru Sakuragi, MD; \\ Makoto Nakahama, MD; Yusuke Kawai, MD; Tadahisa Uesugi, MD; Takefumi Oka, MD; \\ Mitsuru Munemasa, MD; Natsuki Takahashi, MD; Naoki Mukohara, MD; Seiji Habara, MD; \\ Yasushi Koyama, MD; Kazufumi Nakamura, MD; Hiroshi Ito, MD \\ on behalf of the PEACH Investigators
}

\begin{abstract}
Background: The effect of lipid-lowering agents on progression of coronary artery calcification (CAC) remains unclear. We evaluated the effects of pitavastatin $2 \mathrm{mg} /$ day (PIT2), pitavastatin $4 \mathrm{mg} /$ day (PIT4), and PIT2 combined with eicosapentaenoic acid (PIT2+EPA) on CAC progression.

Methods and Results: This prospective multicenter study in Japan included patients with an Agatston score of 1-999, hypercholesterolemia, and no evidence of cardiovascular disease. Patients were allocated into PIT2, PIT4, or PIT2+EPA groups. The primary outcome was the annual percent change in Agatston score in all patients. In total, 156 patients who had multi-detector row computed tomography without any artifacts were included in the primary analysis. Pitavastatin did not significantly reduce the annual progression rate of the Agatston score (40\%; 95\% Cl: 19-61\%). The annual progression rate of Agatston score in the PIT2 group was not significantly different from that in the PIT4 group (34\% vs. $42 \%$, respectively; $\mathrm{P}=0.88$ ) or the PIT2+EPA group ( $34 \%$ vs. $44 \%$, respectively; $\mathrm{P}=0.80$ ). On post-hoc analysis the baseline ratio of low- to high-density lipoprotein cholesterol was a significant predictor of non-progression of Agatston score by pitavastatin (OR, 2.17; 95\% Cl: 1.10-44.12; $\mathrm{P}=0.02)$.
\end{abstract}

Conclusions: Pitavastatin does not attenuate progression of CAC. Intensive pitavastatin treatment and standard treatment with EPA does not reduce progression of CAC compared with standard treatment.

Key Words: Calcification; Computed tomography; Eicosapentaenoic acid; Statin

T he prevalence of coronary artery calcification (CAC) is age and sex dependent, occurring in $\geq 90 \%$ of men and $\geq 67 \%$ of women aged $>70$ years. ${ }^{1,2} \mathrm{CAC}$ is correlated with the degree of atherosclerosis and the rate of future cardiac events. ${ }^{2}$ Several studies have examined whether medical therapy can halt or even reverse progression of CAC. Studies on the effects of statins on progression of CAC have yielded conflicting results, with initial studies showing significant regression of CAC and contemporaneous data showing opposite results. ${ }^{36}$ Several randomized controlled clinical trials have failed to show attenuation of progression of CAC despite a significant lowering of low-density lipoprotein cholesterol (LDL-C) by atorvastatin or pravastatin. ${ }^{7-11}$ Pitavastatin has been reported to substantially reduce LDL-C, as have other statins. ${ }^{12}$ A previous study showed a significant increase in

Received April 19, 2017; revised manuscript received July 12, 2017; accepted July 23, 2017; released online September 1, 2017 Time for primary review: 20 days

Department of Cardiovascular Medicine, Okayama University Graduate School of Medicine, Dentistry and Pharmaceutical Sciences, Okayama (T.M., K.K., K.N., H.I.); Department of Cardiology, Kasaoka Daiichi Hospital, Kasaoka (H.A.); Department of Cardiovascular Medicine, Iwakuni Clinical Center, Iwakuni (S.S.); Department of Cardiology, Fukuyama City Hospital, Fukuyama (M.N.); Department of Cardiology, Ehime Prefectural Center Hospital, Matsuyama (Y. Kawai); Department of Cardiology, Sumitomo Besshi Hospital, Niihama (T.U.); Department of Cardiology, Tsuyama Chuo Hospital, Okayama (T.O.); Department of Cardiology, Okayama Clinical Center, Okayama (M.M.); Department of Cardiology, Matsuyama Shimin Hospital, Matsuyama (N.T.); Department of Cardiology, Himeji Red Cross Hospital, Himeji (N.M.); Department of Cardiology, Kurashiki Central Hospital, Kurashiki (S.H.); and Department of Cardiology, Sakurabashi Watanabe Hospital, Osaka (Y. Koyama), Japan

Mailing address: Toru Miyoshi, MD, PhD, Department of Cardiovascular Medicine, Okayama University, 2-5-1 Shikata-cho, Kita-ku, Okayama 700-8558, Japan. E-mail: miyoshit@cc.okayama-u.ac.jp

ISSN-1346-9843 All rights are reserved to the Japanese Circulation Society. For permissions, please e-mail: cj@j-circ.or.jp 


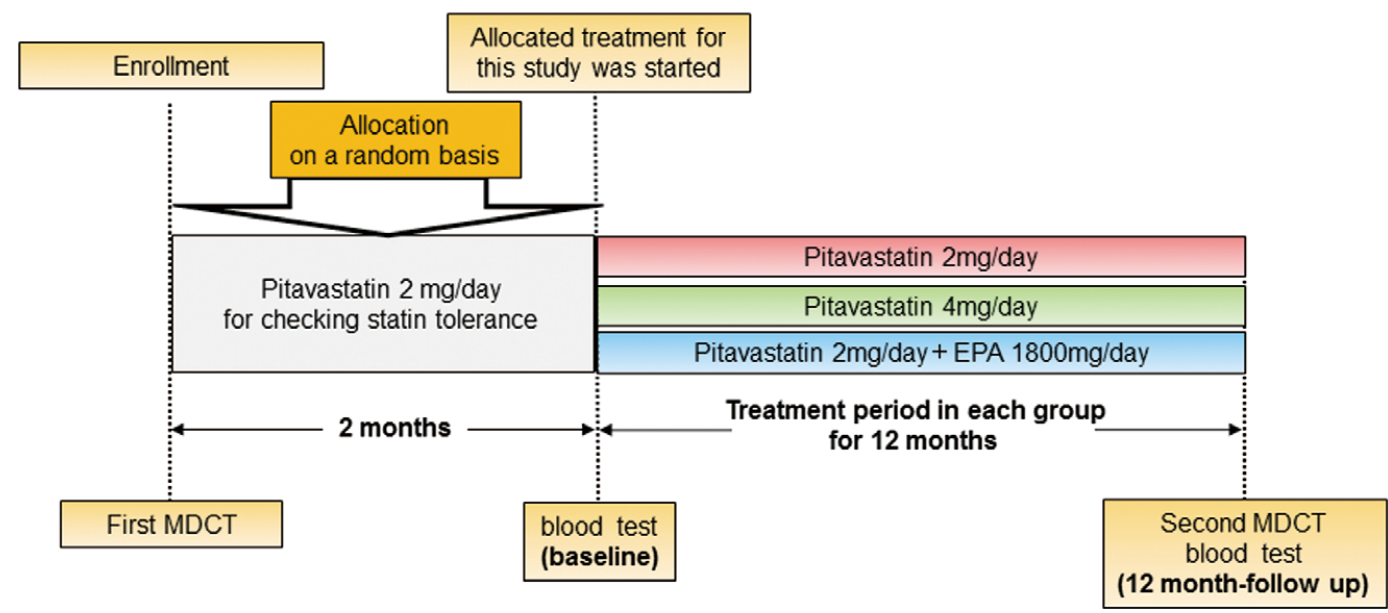

Figure 1. Overview of the study design. Patients were enrolled after determination of the first coronary artery calcification score in each facility. One year after the end of the allocated treatment phase, coronary artery calcification score was redetermined. EPA, eicosapentaenoic acid; MDCT, multi-detector row computed tomography.

high-density lipoprotein cholesterol (HDL-C) after switching from other statins to pitavastatin. ${ }^{13}$ Therefore, pitavastatin has the potential to influence progression of CAC, unlike other statins.

Intake of $\omega-3$ polyunsaturated fatty acids, including eicosapentaenoic acid (EPA) and docosahexaenoic acid, may reduce the risk of fatal cardiovascular disease. ${ }^{14}$ Recent studies have shown that bioactive derivatives of $\omega-3$ polyunsaturated fatty acids, such as resolvins and protectins, reduce inflammation. ${ }^{15}$ Previous studies also demonstrated that early initiation of EPA after revascularization in patients with acute myocardial infarction reduced the acute inflammatory response. ${ }^{16}$ The effect of EPA treatment on CAC, however, remains unknown. Furthermore, the effect of intervention with EPA supplementation on progression of CAC has not been studied.

In the current study, we investigated the annual progression of CAC in patients with hypercholesterolemia who were asymptomatic for cardiovascular disease, and compared the effects of pitavastatin $2 \mathrm{mg}$ /day (PIT2), pitavastatin $4 \mathrm{mg}$ /day (PIT4), and PIT2 combined with EPA $1,800 \mathrm{mg} /$ day (PIT2+EPA) on the annual progression of CAC.

\section{Methods}

\section{Patients}

This study was a prospective, open-labeled, multicenter trial. Participants were enrolled at 27 centers from May 2010 to August 2011. The study was approved by the ethics committees of all hospitals. All participants provided written informed consent before they were enrolled. The study was conducted according to the principles expressed in the Declaration of Helsinki. The study is registered at UMIN Clinical Trials Registry (UMIN000003171).

Eligible patients were adults ( $>20$ years old) with Agatston score 1-999, hypercholesterolemia (LDL-C $\geq 140 \mathrm{mg} / \mathrm{dL}$ at screening or taking a statin), and no history of atherosclerotic cardiovascular disease. The exclusion criteria were a history of coronary revascularization, including percutaneous coronary intervention and coronary artery bypass surgery; Agatston score 0 or $>1,000$; familial hypercholesterolemia; use of cyclosporine; and use of lipid-lowering agents excluding statins.

\section{Study Protocol}

The study design is shown in Figure 1. Patients were enrolled after evaluation of eligibility in each institution, including baseline multi-detector row computed tomography (MDCT) acquisition. All patients then began taking pitavastatin $2 \mathrm{mg} /$ day for 2 months to check for tolerance, and Agatston score was evaluated again at the core laboratory. While the patients were taking pitavastatin for 2 months, they were randomly allocated to the PIT2, PIT4, or PIT2+EPA group. Allocation was conducted by the Clinical Trials Unit based at Okayama University via secure website, and was stratified at each of the 27 centers using random permuted blocks. Stratification factors included baseline Agatston score (1-99/100-999) examined at the core laboratory, age $(<70 / \geq 70$ years), sex, presence of diabetes mellitus, and baseline LDL-C $(<110 / \geq 110 \mathrm{mg} / \mathrm{dL})$ measured at the central laboratory. EPA was a highly purified $(>98 \%)$ EPA ethyl ester (ethyl all-cis-5,8,11,14,17eicosapentaenoate). Baseline blood test data were obtained immediately before starting the allocated treatment. MDCT and blood tests were performed again at 1-year follow-up.

We calculated that a sample of 240 patients would provide at least $80 \%$ power to detect a $\geq 20 \%$ decrease in the primary outcome, which was the annual mean progression rate of CAC Agatston score in all groups. This was based on the assumption of natural progression of Agatston score of $30 \%,{ }^{7-11}$ a dropout rate of $>15 \%$, and a probability of type I error of $5 \%$. In this study, $\leq 10 \%$ progression of the primary outcome was considered clinically significant.

The primary outcomes were percent change in Agatston score and $\mathrm{CAC}$ volume between baseline and final determination (score after 12 months of treatment). To avoid problems with multiplicity, we prioritized the Agatston score first and CAC volume second. The secondary out- 


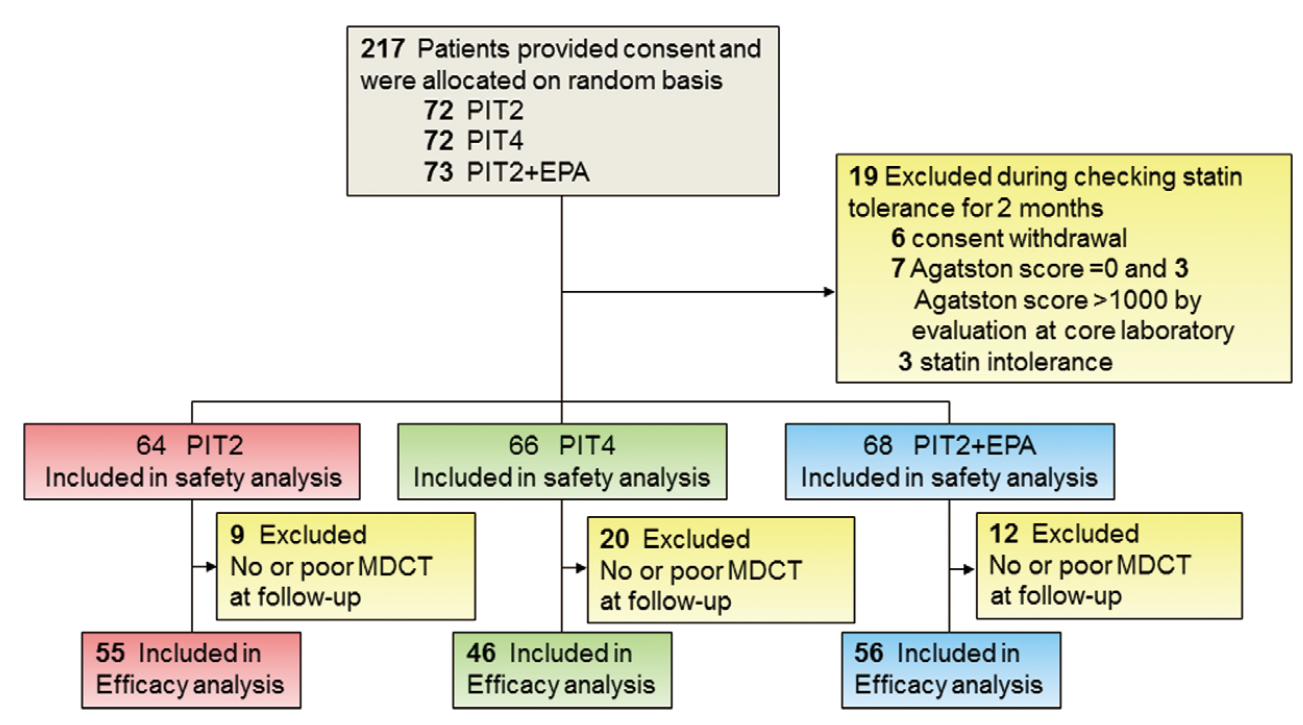

Figure 2. Patient distribution. EPA, eicosapentaenoic acid 1,800 mg/day; MDCT, multi-detector row computed tomography; PIT2, pitavastatin $2 \mathrm{mg} /$ day; PIT4, pitavastatin $4 \mathrm{mg} /$ day.

come measures were the annual changes in the total cholesterol, LDL-C, HDL-C, and triglyceride; EPA/arachidonic acid (EPA/AA) ratio; and high-sensitivity $\mathrm{C}$-reactive protein (hsCRP) level.

\section{MDCT and CAC Analysis}

MDCT was performed in a standardized fashion at all 27 centers. All recruiting sites had previous cardiac CT experience and were equipped with 64-slice or higher advanced CT technology. Electrocardiogram triggering was performed at $80 \%$ of the RR interval. MDCT were documented in a digital imaging and communications in medicine (DICOM) format, which was sent to the core laboratory at L\&L Company (Osaka, Japan) for blinded analysis. The local sites generated the total Agatston score, which was used as the inclusion criterion of this study. The quality of CT was categorized as "good," "moderate" (i.e., not good but readily evaluable), or "not evaluable". For CT with good or moderate quality, CAC was quantified by a qualified CT technologist blinded to all clinical and biochemistry parameters. A calcium threshold of $\geq 130 \mathrm{HU}$ was used. As described by Agatston et al, the calcium score was determined by multiplying the area of each calcified lesion by a weighing factor corresponding to the peak pixel intensity for each lesion. ${ }^{17}$ The sum of each lesion in all of the coronary arteries was used for analysis. The CAC volume score was derived by isotropic interpolation. The calcium volume was calculated by multiplying the number of voxels with the voxel volume using a technique of isotropic interpolation as mentioned by Callister et al. ${ }^{18}$ Image analysis was performed by a trained radiologist and trained cardiologist who were blinded to the patient data. Disagreements in data analysis between the 2 observers were resolved by consensus reading.

\section{Risk Factors and Laboratory Analysis}

We collected information on demographics, smoking status, and medication for each participant. Current smoking was defined as a history of cigarette smoking during the past year. Diabetes was confirmed according to the criteria of the American Diabetes Association ${ }^{19}$ or based on a history of diabetes mellitus treatment. Hypertension was defined as seated blood pressure $\geq 140 / 90 \mathrm{mmHg}$ or undergoing current treatment with anti-hypertensive medication. Previous cardiovascular disease was defined as a documented medical history of cerebrovascular, peripheral arterial, or venous disease; myocardial infarction; angina pectoris; or coronary artery revascularization or positive diagnostic test (ultrasonography, stress test, coronary angiography, or radionuclide imaging). Body mass index was calculated as body weight divided by squared height. Waist circumference was assessed at the umbilicus level, using the mean of 3 measurements.

All laboratory data were measured at an independent central study laboratory (SRL, Tokyo, Japan). Standard enzymatic methods were used to measure total cholesterol, HDL-C, LDL-C, and triglyceride. hsCRP was measured using a Roche-Hitachi assay (Hitachi, Tokyo, Japan).

\section{Safety}

Throughout the study, safety was reported by recording serious adverse events regardless of their causal relationship with the trial drugs, and adverse events of special interest regarding statins and EPA. The investigators reported the incidence of adverse events to the principal investigator. The principal investigator reported this information to the Data and Safety Monitoring Board, which consisted of authorized cardiologists with relevant expertise.

\section{Statistical Analysis}

Efficacy analysis was performed in a modified intention-totreat population, with exclusion of all patients who did not meet the inclusion/exclusion criteria, withdrew consent, did not receive any study treatment, and did not have perfect MDCT without any artifacts. The safety population 


\begin{tabular}{|c|c|c|c|c|c|}
\hline & $\begin{array}{c}\text { PIT2 } \\
(n=55)\end{array}$ & $\begin{array}{c}\text { PIT4 } \\
(n=46)\end{array}$ & $\begin{array}{c}\text { PIT2+EPA } \\
(n=56)\end{array}$ & $\begin{array}{c}\text { Total } \\
(n=157)\end{array}$ & P-value \\
\hline Age (years) & $67 \pm 9$ & $66 \pm 10$ & $68 \pm 9$ & $67 \pm 9$ & 0.42 \\
\hline Male & $29(53)$ & $27(59)$ & $30(54)$ & $86(55)$ & 0.81 \\
\hline BMI $\left(\mathrm{kg} / \mathrm{m}^{2}\right)$ & $25 \pm 4$ & $26 \pm 5$ & $25 \pm 3$ & $25 \pm 4$ & 0.63 \\
\hline Waist circumference $(\mathrm{cm})$ & $89 \pm 10$ & $87 \pm 11$ & $87 \pm 10$ & $88 \pm 10$ & 0.49 \\
\hline SBP $(\mathrm{mmHg})$ & $129 \pm 17$ & $136 \pm 18$ & $133 \pm 19$ & $132 \pm 18$ & 0.13 \\
\hline $\mathrm{DBP}(\mathrm{mmHg})$ & $73 \pm 12$ & $78 \pm 14$ & $74 \pm 10$ & $74 \pm 12$ & 0.11 \\
\hline \multicolumn{6}{|l|}{$\mathrm{CV}$ risk factors } \\
\hline Hypertension & $52(95)$ & $38(83)$ & $38(68)$ & $128(82)$ & 0.004 \\
\hline Diabetes mellitus & $16(29)$ & $13(28)$ & $14(25)$ & $43(27)$ & 0.91 \\
\hline Current or former smoker & $8(15)$ & $9(20)$ & $10(18)$ & $27(17)$ & 0.76 \\
\hline Hemodialysis & $0(0)$ & $1(2)$ & $0(0)$ & $1(1)$ & 0.30 \\
\hline \multicolumn{6}{|l|}{ Baseline Agatston score } \\
\hline $1-99$ & $28(51)$ & $24(52)$ & $27(48)$ & $79(50)$ & 0.73 \\
\hline 100-399 & $18(33)$ & $18(39)$ & $19(34)$ & $55(35)$ & \\
\hline 400-999 & $9(16)$ & $4(9)$ & $10(18)$ & $23(15)$ & \\
\hline \multicolumn{6}{|l|}{ Medication } \\
\hline Anti-hypertensive agents & $51(93)$ & $32(70)$ & $37(66)$ & $120(76)$ & 0.002 \\
\hline Calcium channel blockers & $33(60)$ & $24(52)$ & $22(39)$ & $79(50)$ & 0.09 \\
\hline ACEI & $5(9)$ & $2(4)$ & $1(2)$ & $8(5)$ & 0.21 \\
\hline ARB & $27(49)$ & $19(41)$ & $22(39)$ & $68(43)$ & 0.55 \\
\hline$\beta$-blockers & $14(25)$ & $10(22)$ & $6(11)$ & $30(19)$ & 0.12 \\
\hline Diuretics & $7(13)$ & $4(9)$ & $2(4)$ & $13(8)$ & 0.21 \\
\hline Anti-diabetic agents & $12(22)$ & $7(15)$ & $10(18)$ & $29(18)$ & 0.69 \\
\hline PPAR $y$ agonists & $0(0)$ & $4(9)$ & $3(5)$ & $7(4)$ & 0.10 \\
\hline Metformin & $0(0)$ & $2(4)$ & $3(5)$ & $5(3)$ & 0.24 \\
\hline Sulfonylurea & $3(5)$ & $4(9)$ & $3(5)$ & $10(6)$ & 0.74 \\
\hline$a$-Glucosidase inhibitors & $2(4)$ & $2(4)$ & $3(5)$ & $7(4)$ & 0.91 \\
\hline Insulin use & $2(4)$ & $1(2)$ & $2(4)$ & $5(3)$ & 0.89 \\
\hline
\end{tabular}

Data given as mean \pm SD or $\mathrm{n}(\%)$. There were 3 unknown answers for BMI, 44 for waist circumference, 3 for SBP, 3 for DBP, 2 for hypertension, 1 for diabetes mellitus, 10 for a family history of coronary artery disease, and 1 for smoking. Missing data and unknown answers were excluded from $P$ value calculations. Percentages may not total $100 \%$ because of rounding. ACEI, angiotensin-converting enzyme inhibitor; ARB, angiotensin type 1 receptor blocker; BMI, body mass index; CV, cardiovascular; DBP, diastolic blood pressure; EPA, eicosapentaenoic acid 1,800 mg/day; PIT2, pitavastatin $2 \mathrm{mg} /$ day; PIT4, pitavastatin $4 \mathrm{mg} /$ day; PPAR, peroxisome proliferator-activated receptor; SBP, systolic blood pressure.

was similar to the modified intention-to-treat population except that patients who did not have perfect MDCT images were included. Safety analyses were performed according to the actual treatment received.

Mean Agatston score progression rate and 95\% CI adjusted for the prespecified factors (i.e., baseline Agatston score 1-99/100-999; age <70/ $\geq 70$ years; sex; presence of diabetes mellitus; and baseline LDL-C $<110 / \geq 110 \mathrm{mg} / \mathrm{dL}$ ) were estimated using multivariate linear models. If the upper limit of the confidence interval of the mean Agatston score progression rate was $<30 \%$, we concluded that the primary outcome was met. The same analyses were performed for the $\mathrm{CAC}$ volume score. The mean progression rate of Agatston score in the treatment groups was compared using an analysis of variance model including the same prespecified adjustment factors as described. The Dunnett-Hsu multiple-comparisons method was used for derivation of P-value. The same analysis was performed for the CAC volume score, lipid parameters, and glycemic parameters. For safety analysis, frequencies and percentages were calculated for each adverse event and treatment group. For exploratory analyses, we performed subgroup analyses for the mean progression rate of Agatston score and investigated associations between the incidence of non-progression in Agatston score and baseline lipid parameters using logistic regression models.

Continuous variables are presented as mean \pm SD or median (IQR) as appropriate. Categorical variables are presented as frequency and proportion (\%). Analysis of variance or Kruskal-Wallis test was used to compare continuous variables between the study groups. Chi-squared test was used to compare categorical variables. $\mathrm{P}<0.05$ was considered to indicate statistical significance. All P-values are 2-sided. All analyses were performed using SAS ver. 9.3 (SAS Institute, Cary, NC, USA).

\section{Results}

\section{Study Flow and Baseline Characteristics}

A flow diagram of the study is shown in Figure 2. After screening at 27 sites in Japan, the 217 patients were assigned to the PIT2 group $(n=72)$, the PIT4 group $(n=72)$, or the PIT2+EPA group $(\mathrm{n}=73)$. Nineteen patients who did not meet the inclusion criteria and/or met the exclusion criteria were excluded, and a final total of 198 patients were analyzed as the safety population. Furthermore, 41 


\begin{tabular}{|c|c|c|c|c|}
\hline & $\begin{array}{l}\text { Overall } \\
(n=157)\end{array}$ & $\begin{array}{c}\text { PIT2 } \\
(n=55)\end{array}$ & $\begin{array}{c}\text { PIT4 } \\
(n=46)\end{array}$ & $\begin{array}{c}\text { PIT2+EPA } \\
(n=56)\end{array}$ \\
\hline \multicolumn{5}{|l|}{ Agatston score } \\
\hline \multicolumn{5}{|l|}{ Baseline } \\
\hline Median (IQR) & $97(28-231)$ & $91(28-299)$ & $88(24-200)$ & $108(27-248)$ \\
\hline \multicolumn{5}{|l|}{ Month 12} \\
\hline Median (IQR) & $118(42-264)$ & $120(28-265)$ & $109(40-241)$ & $139(46-301)$ \\
\hline \multicolumn{5}{|l|}{$\%$ Change from baseline } \\
\hline LS Mean $(95 \% \mathrm{Cl})$ & $40(19-61)$ & $34(5-63)$ & $42(9-75)$ & $44(16-73)$ \\
\hline LS Mean difference $(95 \% \mathrm{Cl})$ & & & $8(-34$ to 50$)$ & $10(-30$ to 50$)$ \\
\hline Adjusted P-valuevs. PIT2 & & & 0.88 & 0.80 \\
\hline \multicolumn{5}{|l|}{ CAC volume score $\left(\mathrm{mm}^{3}\right)$} \\
\hline \multicolumn{5}{|l|}{ Baseline } \\
\hline Median (IQR) & $91(27-198)$ & $85(34-250)$ & $81(22-178)$ & $101(27-218)$ \\
\hline \multicolumn{5}{|l|}{ Month 12} \\
\hline Median (IQR) & $116(43-222)$ & $108(36-222)$ & $94(33-216)$ & $130(49-248)$ \\
\hline \multicolumn{5}{|l|}{$\%$ Change from baseline } \\
\hline LS mean $(95 \% \mathrm{Cl})$ & $34(22-47)$ & $30(12-47)$ & $42(22-61)$ & $34(17-51)$ \\
\hline LS mean difference $(95 \% \mathrm{Cl})$ & & & $12(-13$ to 37$)$ & $4(-20$ to 28$)$ \\
\hline Adjusted P-valuevs. PIT2 & & & 0.46 & 0.90 \\
\hline
\end{tabular}

Dunnett-Hsu multiplicity adjusted P-value. CAC, coronary artery calcification; LS, least squares. Other abbreviations as in Table 1.

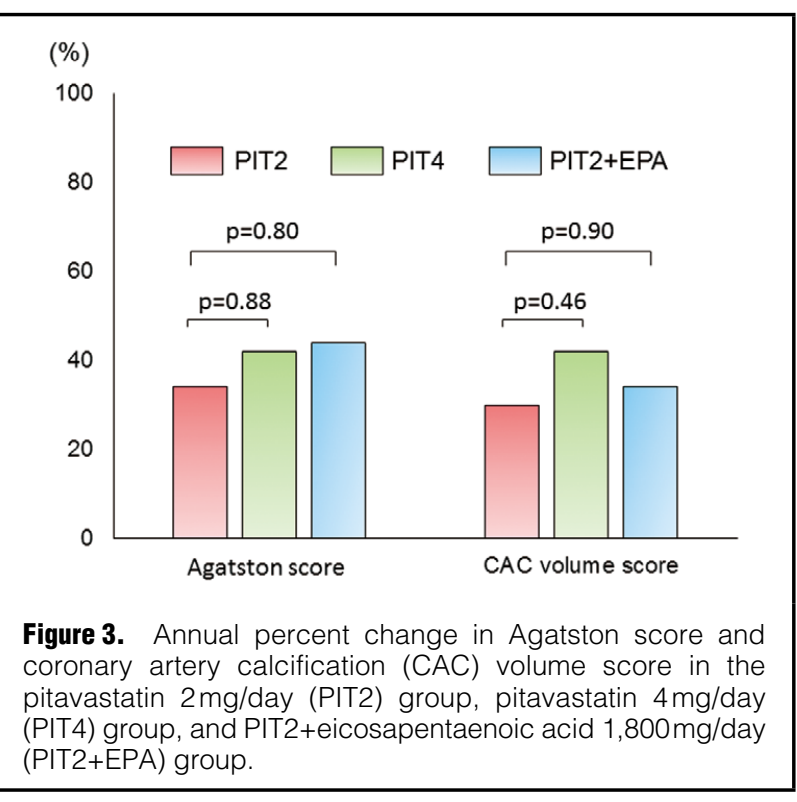

patients were excluded because they had no or poor MDCT at follow-up, and 157 patients (PIT2 group, $n=55$; PIT4 group, $n=46$; and PIT2+EPA group, $n=56$ ) comprised the primary analysis group.

The demographics of the efficacy analysis set are listed in Table 1. The baseline characteristics were well balanced between the groups except for the prevalence of hypertension and use of anti-hypertensive agents. There was no significant difference in serum creatinine (PIT2: median, $0.76 \mathrm{mg} / \mathrm{dL}$; IQR, 0.62-0.90 mg/dL; PIT4: median, $0.80 \mathrm{mg} / \mathrm{dL}$; IQR, 0.69-0.91 mg/dL; PIT2+EPA: median, $0.76 \mathrm{mg} / \mathrm{dL}$; IQR, $0.66-0.84 \mathrm{mg} / \mathrm{dL}$; Kruskal-Wallis test, $\mathrm{P}=0.2553)$. There was also no significant difference in the estimated glomerular filtration rate (PIT2: median, $71.3 \mathrm{~mL} / \mathrm{min} / 1.73 \mathrm{~m}^{2}$; IQR, $59.4-79.0 \mathrm{~mL} / \mathrm{min} / 1.73 \mathrm{~m}^{2}$; PIT4, median, $65.9 \mathrm{~mL} / \mathrm{min} / 1.73 \mathrm{~m}^{2}$; IQR, $61.1-75.2 \mathrm{~mL} / \mathrm{min} / 1.73 \mathrm{~m}^{2}$; PIT2+EPA: median, $68.2 \mathrm{~mL} / \mathrm{min} / 1.73 \mathrm{~m}^{2}$; IQR, $61.5-81.0 \mathrm{~mL} / \mathrm{min} / 1.73 \mathrm{~m}^{2} ;$ Kruskal-Wallis test, $\mathrm{P}=0.5387$ ). Baseline Agatston and CAC volume scores were well balanced $(\mathrm{P}=0.72$ for Agatston score and $\mathrm{P}=0.62$ for $\mathrm{CAC}$ volume score; Table 2). A total of $85 \%$ of all participants had Agatston score $<400$.

\section{Efficacy Analysis of Primary Outcomes}

Figure 3, Table 2 show the primary outcomes. In the overall group, the mean progression rate of Agatston score and of volume score adjusted for baseline CAC score, age, sex, presence of diabetes mellitus, and baseline LDL-C was 40\% (95\% CI: $19-61 \%$ ) and 34\% (95\% CI: $22-47 \%)$, respectively. This indicates that pitavastatin did not significantly reduce the annual Agatston score progression rate. The mean Agatston score progression rate was 34\% (95\% CI: $5-63 \%)$ in the PIT2 group, $42 \%(95 \%$ CI: $9-75 \%)$ in the PIT4 group, and 44\% (95\% CI: $16-73 \%$ ) in the PIT2+EPA group. There was no significant difference in Agatston score progression rate between the 3 groups $(\mathrm{P}=0.88$ for PIT2 vs. PIT4 group, and $\mathrm{P}=0.80$ for PIT2 vs. PIT2+EPA group). The mean progression rate of the CAC volume was $30 \%$ (95\% CI: $12-47 \%)$ in the PIT2 group, $42 \%$ (95\% CI: $22-61 \%)$ in the PIT4 group, and $34 \%(95 \%$ CI: $17-51 \%)$ in the PIT2+EPA group. There was also no significant difference in the progression rate of the CAC volume scores between the 3 groups ( $\mathrm{P}=0.46$ for PIT 2 vs. PIT 4 group and $\mathrm{P}=0.90$ for PIT2 vs. PIT2+EPA group).

We also assessed the difference in the use of anti-hypertensive drugs between the groups. Therefore, an exploratory variable of anti-hypertensive drug use was added to the primary analysis model. In the overall group, the adjusted mean Agatston score progression rate was $45 \%$ (95\% CI: 22-68\%). The mean Agatston score progression rate by group was $41 \%$ (95\% CI: $8-74 \%$ ) in the PIT2 group, 


\begin{tabular}{|c|c|c|c|c|}
\hline & $\begin{array}{l}\text { Overall } \\
(n=157)\end{array}$ & $\begin{array}{c}\text { PIT2 } \\
(n=55)\end{array}$ & $\begin{array}{c}\text { PIT4 } \\
(n=46)\end{array}$ & $\begin{array}{c}\text { PIT2+EPA } \\
(n=56)\end{array}$ \\
\hline \multicolumn{5}{|c|}{ Percent change from baseline } \\
\hline Overall & $36(22-51), 157$ & $31(2-60), 55$ & 38 (13-63), 46 & 40 (17-64), 56 \\
\hline \multicolumn{5}{|l|}{ Gender } \\
\hline Female & 35 (11-60), 71 & 46 ( -13 to 105$), 26$ & $18(1-36), 19$ & $37(2-73), 26$ \\
\hline Male & 37 (19-55), 86 & 18 (-0 to 36$), 29$ & $52(10-94), 27$ & 43 (10-75), 30 \\
\hline \multicolumn{5}{|c|}{ Age (years) } \\
\hline$\geq 70$ & 44 (20-69), 66 & 51 ( -13 to 115$), 23$ & 37 (17-57), 17 & 43 (15-72), 26 \\
\hline$<70$ & 31 (12-49), 91 & $17(-5$ to 38$), 32$ & 39 ( -0 to 78$), 29$ & 38 (0-75), 30 \\
\hline \multicolumn{5}{|c|}{ Agatston score } \\
\hline$\geq 100$ & 22 (17-28), 78 & 19 (9-28), 27 & 31 (19-42), 22 & 19 (11-27), 29 \\
\hline$<100$ & 50 (22-79), 79 & 43 (-14 to 100$), 28$ & 45 ( -4 to 94$), 24$ & 63 (15-111), 27 \\
\hline \multicolumn{5}{|c|}{ LDL-C (mg/dL) } \\
\hline$\geq 110$ & 33 ( -2 to 68$), 29$ & 18 (-25 to 60$), 10$ & 18 (-39 to 75$), 6$ & 52 (-22 to 126$), 13$ \\
\hline$<110$ & $37(21-54), 127$ & 34 (-0 to 69$), 45$ & 42 (13-71), 39 & $37(14-60), 43$ \\
\hline \multicolumn{5}{|c|}{ Diabetes mellitus } \\
\hline Yes & $43(12-75), 43$ & $28(-12$ to 68$), 16$ & 59 (-31 to 149$), 13$ & $47(4-90), 14$ \\
\hline No & $34(17-51), 114$ & $33(-6$ to 71$), 39$ & 30 (16-44), 33 & $38(9-67), 42$ \\
\hline \multicolumn{5}{|c|}{ Hypertension } \\
\hline Yes & 32 (17-47), 128 & 30 ( -1 to 60$), 52$ & 25 (12-37), 38 & 42 (15-70), 38 \\
\hline No & 56 (9-103), 29 & 57 (-116 to 229$), 3$ & 101 ( -51 to 253$), 8$ & 36 (-13 to 84$), 18$ \\
\hline
\end{tabular}

There was no significant difference in the progression rate of the Agatston scores among the 3 groups within each subgroup. LDL-C, lowdensity lipoprotein cholesterol. Other abbreviations as in Table 1.

$45 \%(95 \%$ CI: $12-79 \%)$ in the PIT4 group, and $47 \%(95 \%$ CI: $18-76 \%)$ in the PIT2+EPA group. There was no significant difference in Agatston score progression rate between the 3 groups $(\mathrm{P}=0.97$ for PIT2 vs. PIT4 and $\mathrm{P}=0.94$ for $\mathrm{PIT} 2$ vs. PIT2+EPA).

\section{Serial Lipid Changes and Glycemic Parameters}

Table 3 lists the serial changes in lipid parameters and hsCRP measured at the core laboratory from the start of the allocated treatment to 12-month follow-up. When the allocated treatments were started, LDL-C in all groups was $<100 \mathrm{mg} / \mathrm{dL}$ because of the use of pitavastatin at $2 \mathrm{mg} /$ day for 2 months to check statin tolerance. After 12 months of treatment, the PIT4 group had a further reduction in LDL-C compared with the PIT2 group. In the PIT2+EPA group, triglyceride was significantly reduced. After 12 months of treatment, hemoglobin Alc (HbAlc) was significantly reduced only in the PIT4 group. EPA/AA ratio was increased only in the PIT2+EPA group. No significant changes in hsCRP were observed in the PIT4 or PIT2+EPA groups. Table S1 lists the changes in the lipid parameters from the time of enrollment to the 12-month follow-up as measured at each institute, not at the core laboratory. LDL-C was significantly decreased in all groups. HDL-C in the PIT4 group was significantly increased.

\section{Safety Analysis}

A summary of adverse events is given in Table S2. The overall number of adverse events during the allocated treatment phase was similar between the 3 groups. No patients developed rhabdomyolysis. No cardiovascular events, including cardiovascular death, non-fatal myocardial infarction, and non-fatal stroke, were observed during the study period.

\section{Exploratory Analyses}

Table 3 lists the results of the subgroup analysis. There was no significant difference in Agatston score progression rate within any subgroup, between the 3 groups. We also investigated associations between the incidence of Agatston score non-progression and baseline lipid parameters using logistic regression models (Table 4). In this study, Agatston score non-progression was defined as $\leq 0 \%$ change from baseline in Agatston score. In all 3 groups, patients with higher baseline LDL-C and/or LDL-C/HDL-C ratio had significantly higher odds of non-progression of Agatston score. In the PIT4 group, patients with a higher baseline LDL-C/HDL-C ratio had higher odds of non-progression. In the PIT2+EPA group, a higher baseline total cholesterol, HDL-C, and/or LDL-C had a significant effect on non-progression.

\section{Discussion}

In this study, we found that the annual progression rate of CAC while receiving pitavastatin was $>30 \%$ in patients with hypercholesterolemia who were asymptomatic for cardiovascular disease. Additionally, progression of CAC was not attenuated in the PIT4 group or PIT2+EPA group compared with the PIT2 group. To the best of our knowledge, this is the first prospective study to report the annual progression rate of CAC with pitavastatin use in a Japanese population and to compare the effect of the statin dose or a statin combined with EPA on the progression of CAC.

The annual progression rate of the Agatston score and CAC volume score in the PIT2 group was $>30 \%$ in this study. This is greater than expected according to previous randomized trials, with results ranging from approximately $15 \%$ to $27 \%$ per year. ${ }^{7-11}$ One explanation for this 


\begin{tabular}{|c|c|c|c|c|}
\hline & $\begin{array}{l}\text { Overall } \\
(n=157)\end{array}$ & $\begin{array}{c}\text { PIT2 } \\
(n=55)\end{array}$ & $\begin{array}{l}\text { PIT4 }{ }^{\ddagger} \\
(n=45)\end{array}$ & $\begin{array}{c}\text { PIT2+EPA } \\
(n=56)\end{array}$ \\
\hline \multicolumn{5}{|l|}{$\begin{array}{l}\text { Incidence of non-progression in } \\
\text { Agatston score, } \mathrm{n}(\%)\end{array}$} \\
\hline \multicolumn{5}{|l|}{ Improvement } \\
\hline Yes & $39(25)$ & $15(27)$ & $10(22)$ & $14(25)$ \\
\hline No & $118(75)$ & $40(73)$ & $36(78)$ & $42(75)$ \\
\hline \multicolumn{5}{|c|}{$\begin{array}{l}\text { Non-progression in Agatston score and } \\
\text { baseline lipid parameters }\end{array}$} \\
\hline \multicolumn{5}{|c|}{ Total cholesterol (mg/dL) } \\
\hline OR $(95 \% \mathrm{Cl})$ per $10 \mathrm{mg} / \mathrm{dL}$ & $1.01(1.00-1.02)$ & $1.00(0.98-1.02)$ & $1.00(0.98-1.03)$ & $1.03(1.00-1.05)$ \\
\hline P-value & 0.0925 & 0.8935 & 0.8565 & 0.0181 \\
\hline \multicolumn{5}{|l|}{ LDL-C (mg/dL) } \\
\hline OR $(95 \% \mathrm{Cl})$ per $10 \mathrm{mg} / \mathrm{dL}$ & $1.02(1.00-1.03)$ & $1.01(0.98-1.03)$ & $1.01(0.98-1.05)$ & $1.03(1.01-1.06)$ \\
\hline P-value & 0.0191 & 0.6198 & 0.4013 & 0.0179 \\
\hline \multicolumn{5}{|l|}{ HDL-C (mg/dL) } \\
\hline OR $(95 \% \mathrm{Cl})$ per $10 \mathrm{mg} / \mathrm{dL}$ & $0.99(0.96-1.02)$ & $0.97(0.92-1.01)$ & $0.95(0.89-1.02)$ & $1.07(1.01-1.13)$ \\
\hline P-value & 0.4715 & 0.1525 & 0.1367 & 0.0334 \\
\hline \multicolumn{5}{|l|}{ Triglyceride (mg/dL) } \\
\hline OR $(95 \% \mathrm{Cl})$ per $10 \mathrm{mg} / \mathrm{dL}$ & $1.00(0.99-1.00)$ & $1.00(1.00-1.01)$ & $1.00(0.99-1.01)$ & $0.99(0.98-1.00)$ \\
\hline P-value & 0.8214 & 0.5296 & 0.5101 & 0.1912 \\
\hline \multicolumn{5}{|l|}{$\mathrm{L} / \mathrm{H}$} \\
\hline OR $(95 \% \mathrm{Cl})$ & $2.17(1.14-4.12)$ & $2.04(0.76-5.52)$ & $4.26(1.06-17.15)$ & $1.47(0.50-4.31)$ \\
\hline P-value & 0.0181 & 0.1592 & 0.0413 & 0.4845 \\
\hline \multicolumn{5}{|l|}{ EPA/AA } \\
\hline OR $(95 \% \mathrm{Cl})$ & $1.70(0.43-6.70)$ & $2.44(0.28-21.46)$ & $0.70(0.02-26.64)$ & $1.56(0.20-12.26)$ \\
\hline P-value & 0.4482 & 0.4208 & 0.8500 & 0.6710 \\
\hline \multicolumn{5}{|l|}{$\mathrm{HbA1c}$} \\
\hline OR $(95 \% \mathrm{Cl})$ & $0.86(0.46-1.59)$ & $0.80(0.29-2.18)$ & $0.68(0.24-1.96)$ & $1.51(0.36-6.34)$ \\
\hline P-value & 0.6261 & 0.6588 & 0.8500 & 0.5745 \\
\hline
\end{tabular}

${ }^{\dagger} \leq 0 \%$ change from baseline in Agatston score. $¥$ One missing data point at baseline, and this was excluded from the analyses. AA, arachidonic acid; HbA1c, hemoglobin A1c; HDL-C, high-density lipoprotein cholesterol; L/H, low-density lipoprotein/high-density lipoprotein. Other abbreviations as in Tables $1-3$.

discrepancy is the differences in ethnicity and risk factors among the participants. In a multi-ethnic study of atherosclerosis, black or Hispanic ethnicity was a strong negative factor associated with progression of CAC compared with white ethnicity. ${ }^{20}$ Additionally, among conventional atherosclerotic risk factors, significant risk factors for CAC acceleration are age, male sex, hypertension, and diabetes mellitus. The present study included patients with advanced age (mean age, 67 years), and a high proportion of patients had hypertension $(82 \%)$ and diabetes $(27 \%)$. These factors may affect changes in CAC.

Progression of Agatston score is an independent factor associated with cardiovascular events. Budoff et al found that a $>15 \%$ yearly increase in Agatston score added a significant incremental value over the baseline value, time between scans, and demographic characteristics in terms of predicting all-cause mortality. ${ }^{21}$ Additionally, progression of Agatston score is related to several traditional modifiable and non-modifiable cardiovascular risk factors. ${ }^{22}$ Recent studies have shown, however, that aggressive lipid lowering with high-dose statins promotes coronary calcification. ${ }^{23,24}$ Thus, the clinical significance of progression of coronary calcification remains controversial. In agreement with previous studies on statins,,${ }^{4,9,10}$ progression of Agatston score in the present PIT4 group was greater than that in the PIT2 group. Statins may affect plaque repair and healing by active replacement of the lipid core with fibrosis and calcification. ${ }^{25}$ The higher doses of statins may have accelerated CAC progression in the present study. We also found a greater increase in the Agatston score in the PIT2+EPA than PIT2 group. To our knowledge, this is the first prospective study to investigate the influence of EPA treatment on progression of CAC. Previous clinical studies have shown that a low EPA/AA ratio is associated with formation of high-risk coronary plaques. ${ }^{26}$ EPA reduces the content of $\mathrm{AA}$ in membrane phospholipids, resulting in a competitive decrease in the production of AA-derived pro-inflammatory mediators. ${ }^{27}$ Additionally, conversion of $\omega-3$ polyunsaturated fatty acids into oxygenated bioactive derivatives, such as resolvins and protectins, reduces inflammation. ${ }^{\mathbf{1 5 , 2 8}-\mathbf{3 0}}$ These anti-inflammatory effects of EPA could induce plaque stabilization. The direct effect of EPA on CAC progression, however, remains unknown. We previously reported that a low EPA dose was associated with greater calcification in patients with acute myocardial infarction..$^{31}$ One experimental study reported that N-3 fatty acids inhibit vascular calcification.32 Although the mechanism of the effect of EPA combined with a statin on CAC has not been fully elucidated, EPA might augment the effect of pitavastatin on CAC progression. Further basic and clinical studies are required to clarify the effect of EPA with or without statins on CAC progression. 
No cardiovascular events occurred during the study period. In a multicenter prospective randomized trial using intravascular ultrasound, treatment with pitavastatin at 4 $\mathrm{mg}$ for $8-12$ months significantly decreased plaque volume. ${ }^{33}$ The clinical significance of changes in CAC with respect to an underlying shift in plaque volume remains unknown. In terms of CAC, spotty calcification and dense calcium may have different effects on clinical outcome. ${ }^{34}$ Future discrimination between these 2 completely different sources of coronary calcium might provide a breakthrough in CAC imaging because spotty calcification is a marker of high-risk plaque. ${ }^{34}$

In the present study, LDL-C significantly changed in the PIT4 group but not in the PIT2 group. Baseline data were collected after the patients had taken pitavastatin at $2 \mathrm{mg} /$ day for 2 months to check tolerability. This explains why there were no differences in LDL-C between baseline and the end of the study in the PIT2 group (PIT2 vs. PIT4 group and PIT2 vs. PIT2+EPA group). Compliance was checked by questionnaire: the percentage of participants who took the study drugs for $>5$ days per week was $100 \%$ in the PIT2 group, $89 \%$ in the PIT4 group, and $96 \%$ in PIT2+EPA group. A phase III study in Europe showed a mean percent reduction in LDL-C of $38 \%$ and $45 \%$ following 12 weeks of pitavastatin $2 \mathrm{mg}(\mathrm{n}=315)$ and $4 \mathrm{mg}(\mathrm{n}=298)$, respectively. ${ }^{35}$ These results were similar to that of the control groups taking atorvastatin $10 \mathrm{mg}$ and $20 \mathrm{mg} \cdot{ }^{35}$ Therefore, pitavastatin has sufficient efficacy for lowering LDL-C.

The risk of incident diabetes in patients treated with statins has been gaining attention. ${ }^{36}$ In the current study, the HbA1c level in the PIT4 group was significantly reduced after 12 months of treatment. Huang et al reported a decrease in HbAlc after 6 months of pitavastatin treatment in 222 patients with type 2 diabetes whose anti-diabetic agents were not changed. ${ }^{37}$ The present $\mathrm{HbAlc}$ results are consistent with those of previous studies. ${ }^{37-39}$ In addition, the change in HDL-C from the time of enrollment to 12-month follow-up, as measured at each institute, was significantly increased in the PIT4 group. The influence of these changes on CAC has not been clearly determined, but changes in these parameters may be favorable for cardiovascular events.

\section{Study Limitations}

This study has some limitations. The first limitation was the failure to enroll the target number of patients (214 of 240). The predefined enrollment period was extended once because of the strict entry criteria. The steering committee, however, reluctantly concluded that enrollment had to terminate before enrolling 240 patients because of the limited budget. Future studies should thus involve a larger number of institutes and a longer study period. Second, this study was open labeled, which may have led to potentially biased results. Third, the patients were randomly allocated to 3 groups, while $9 \%$ of the enrolled patients were excluded before the start of the allocated treatment. Furthermore, patients with no or poor MDCT at followup were also excluded. These factors may have decreased homogeneity among the 3 groups. Although a prespecified adjustment for the primary endpoint was performed, the results should be interpreted with caution. Fourth, the planned sample size was not achieved and the study was underpowered. The estimated effect size of the primary outcome, however, was opposite to that expected $(>30 \%)$, which may have mitigated the influence of the low-powered statistical test on the conclusion of the study. Fifth, the duration of follow-up was set at 1 year based on prior experience. ${ }^{7-11}$ If a longer follow-up period had been used, a difference in the effects of the study drug on progression of CAC might have been seen. Finally, data on coronary CT angiography were not available in this study. Therefore, changes in the plaque volume and morphology could not be evaluated. Further investigation is warranted to address this matter.

\section{Conclusions}

CAC increased by $>30 \%$ annually in a group of Japanese patients with hypercholesterolemia who were asymptomatic for cardiovascular disease. Treatment with high-dose pitavastatin and pitavastatin with EPA was unable to attenuate progression of CAC compared with pitavastatin at $2 \mathrm{mg} /$ day.

\section{Acknowledgments}

We thank Yusuke Katayama, MD, Ritsuko Terasaka, MD, Atsushi Mima, MD, Hitoshi Matsubara, MD, Shingo Hosogi, MD, Masayuki Doi, MD, Masayuki Ueeda, MD, Norio Urabe, MD, Kazufumi Takeuchi, MD, Yasuharu Namba, MD, Tetsuya Sato, MD, Nobuyuki Yamada, MD, Masahito Taniguchi, MD, Yutaka Kajikawa, MD, Kouki Watanabe, MD, Kenichi Hisamatsu, MD, Hiroo Kobayashi, MD, and Kiyoaki Maekawa, MD, who were the PEACH investigators. We thank Kaoru Akazawa, Miyuki Fujiwara, and Masayo Ohmori for their technical assistance.

\section{Disclosures}

T.M. received an honorarium from Mochida Pharmaceutical. H.I. received honoraria from Kowa Pharmaceutical and Mochida Pharmaceutical. The other authors declare no conflicts of interest.

\section{Grant Support}

This study was funded by the Japan Heart Foundation (No. 12090021)

\section{References}

1. Maahs DM, Snell-Bergeon JK, Kinney GL, Wadwa RP, Garg $\mathrm{S}$, Ogden LG, et al. ACE-I/ARB treatment in type 1 diabetes patients with albuminuria is associated with lower odds of progression of coronary artery calcification. J Diabetes Complications 2007; 21: 273-279.

2. Madhavan MV, Tarigopula M, Mintz GS, Maehara A, Stone GW, Genereux P. Coronary artery calcification: Pathogenesis and prognostic implications. J Am Coll Cardiol 2014; 63: 1703 -1714.

3. Callister TQ, Raggi P, Cooil B, Lippolis NJ, Russo DJ. Effect of HMG-CoA reductase inhibitors on coronary artery disease as assessed by electron-beam computed tomography. N Engl J Med 1998; 339: $1972-1978$.

4. Achenbach S, Ropers D, Pohle K, Leber A, Thilo C, Knez A, et al. Influence of lipid-lowering therapy on the progression of coronary artery calcification: A prospective evaluation. Circulation 2002; 106: 1077-1082.

5. Budoff MJ, Lane KL, Bakhsheshi H, Mao S, Grassmann BO, Friedman BC, et al. Rates of progression of coronary calcium by electron beam tomography. Am J Cardiol 2000; 86: 8-11.

6. Hecht HS, Harman SM. Relation of aggressiveness of lipidlowering treatment to changes in calcified plaque burden by electron beam tomography. Am J Cardiol 2003; 92: 334-336.

7. Arad Y, Spadaro LA, Roth M, Newstein D, Guerci AD. Treatment of asymptomatic adults with elevated coronary calcium scores with atorvastatin, vitamin $\mathrm{C}$, and vitamin E: The St. Francis Heart Study randomized clinical trial. J Am Coll Cardiol 2005; 46: $166-172$.

8. Raggi P, Davidson M, Callister TQ, Welty FK, Bachmann GA, Hecht $\mathrm{H}$, et al. Aggressive versus moderate lipid-lowering therapy in hypercholesterolemic postmenopausal women: Beyond Endorsed Lipid Lowering with EBT Scanning (BELLES). Circu- 
lation 2005; 112: $563-571$.

9. Houslay ES, Cowell SJ, Prescott RJ, Reid J, Burton J, Northridge DB, et al. Progressive coronary calcification despite intensive lipid-lowering treatment: A randomised controlled trial. Heart 2006; 92: 1207-1212.

10. Schmermund A, Achenbach S, Budde T, Buziashvili Y, Forster A, Friedrich G, et al. Effect of intensive versus standard lipidlowering treatment with atorvastatin on the progression of calcified coronary atherosclerosis over 12 months: A multicenter, randomized, double-blind trial. Circulation 2006; 113: 427-437.

11. Terry JG, Carr JJ, Kouba EO, Davis DH, Menon L, Bender K, et al. Effect of simvastatin $(80 \mathrm{mg})$ on coronary and abdominal aortic arterial calcium (from the coronary artery calcification treatment with zocor [CATZ] study). Am J Cardiol 2007; 99: $1714-1717$.

12. Alagona P Jr. Pitavastatin: Evidence for its place in treatment of hypercholesterolemia. Core Evid 2010; 5: 91-105.

13. Teramoto T, Shimano H, Yokote K, Urashima M. Effects of pitavastatin (LIVALO tablet) on high density lipoprotein cholesterol (HDL-C) in hypercholesterolemia. $J$ Atheroscler Thromb 2009; 16: 654-661.

14. Kimmig LM, Karalis DG. Do omega-3 polyunsaturated fatty acids prevent cardiovascular disease?: A review of the randomized clinical trials. Lipid Insights 2013; 6: 13-20.

15. Serhan CN. Novel lipid mediators and resolution mechanisms in acute inflammation: To resolve or not? Am J Pathol 2010; 177: $1576-1591$.

16. Doi M, Nosaka K, Miyoshi T, Iwamoto M, Kajiya M, Okawa $\mathrm{K}$, et al. Early eicosapentaenoic acid treatment after percutaneous coronary intervention reduces acute inflammatory responses and ventricular arrhythmias in patients with acute myocardial infarction: A randomized, controlled study. Int J Cardiol 2014; 176: $577-582$.

17. Agatston AS, Janowitz WR, Hildner FJ, Zusmer NR, Viamonte M Jr, Detrano R. Quantification of coronary artery calcium using ultrafast computed tomography. J Am Coll Cardiol 1990; 15: $827-832$.

18. Callister TQ, Cooil B, Raya SP, Lippolis NJ, Russo DJ, Raggi P. Coronary artery disease: Improved reproducibility of calcium scoring with an electron-beam CT volumetric method. Radiology 1998; 208: 807-814.

19. American Diabetes Association. Standards of medical care in diabetes-2008. Diabetes Care 2008; 31(Suppl 1): S12-S54.

20. Gassett AJ, Sheppard L, McClelland RL, Olives C, Kronmal R, Blaha MJ, et al. Risk factors for long-term coronary artery calcium progression in the multi-ethnic study of atherosclerosis. $J$ Am Heart Assoc 2015; 4: e001726.

21. Budoff MJ, Hokanson JE, Nasir K, Shaw LJ, Kinney GL, Chow $\mathrm{D}$, et al. Progression of coronary artery calcium predicts all-cause mortality. JACC Cardiovasc Imaging 2010; 3: 1229-1236.

22. Kronmal RA, McClelland RL, Detrano R, Shea S, Lima JA, Cushman M, et al. Risk factors for the progression of coronary artery calcification in asymptomatic subjects: Results from the Multi-Ethnic Study of Atherosclerosis (MESA). Circulation 2007; 115: 2722-2730.

23. Puri R, Nicholls SJ, Shao M, Kataoka Y, Uno K, Kapadia SR, et al. Impact of statins on serial coronary calcification during atheroma progression and regression. J Am Coll Cardiol 2015; 65: $1273-1282$.

24. Henein M, Granasen G, Wiklund U, Schmermund A, Guerci A, Erbel R, et al. High dose and long-term statin therapy accelerate coronary artery calcification. Int J Cardiol 2015; 184: 581-586.

25. Zhao XQ, Yuan C, Hatsukami TS, Frechette EH, Kang XJ, Maravilla KR, et al. Effects of prolonged intensive lipid-lowering therapy on the characteristics of carotid atherosclerotic plaques in vivo by MRI: A case-control study. Arterioscler Thromb Vasc Biol 2001; 21: 1623-1629.
26. Urabe Y, Yamamoto H, Kitagawa T, Utsunomiya H, Tsushima $\mathrm{H}$, Tatsugami F, et al. Association between serum levels of n-3 polyunsaturated fatty acids and coronary plaque detected by coronary computed tomography angiography in patients receiving statin therapy. Circ J 2013; 77: 2578-2585.

27. Calder PC. N-3 polyunsaturated fatty acids and inflammation: From molecular biology to the clinic. Lipids 2003; 38: 343-352.

28. Serhan CN, Clish CB, Brannon J, Colgan SP, Chiang N, Gronert $\mathrm{K}$. Novel functional sets of lipid-derived mediators with antiinflammatory actions generated from omega-3 fatty acids via cyclooxygenase 2-nonsteroidal antiinflammatory drugs and transcellular processing. $J$ Exp Med 2000; 192: 1197-1204.

29. Bannenberg GL, Chiang N, Ariel A, Arita M, Tjonahen E, Gotlinger KH, et al. Molecular circuits of resolution: Formation and actions of resolvins and protectins. J Immunol 2005; 174: $4345-4355$.

30. Arnold C, Markovic M, Blossey K, Wallukat G, Fischer R, Dechend R, et al. Arachidonic acid-metabolizing cytochrome P450 enzymes are targets of \{omega $\}-3$ fatty acids. J Biol Chem 2010; 285: 32720-32733.

31. Ueeda M, Doumei T, Takaya Y, Shinohata R, Katayama Y, Ohnishi N, et al. Serum N-3 polyunsaturated fatty acid levels correlate with the extent of coronary plaques and calcifications in patients with acute myocardial infarction. Circ J 2008; 72: $1836-1843$.

32. Abedin M, Lim J, Tang TB, Park D, Demer LL, Tintut Y. N-3 fatty acids inhibit vascular calcification via the p38-mitogenactivated protein kinase and peroxisome proliferator-activated receptor-gamma pathways. Circ Res 2006; 98: 727-729.

33. Hiro T, Kimura T, Morimoto T, Miyauchi K, Nakagawa Y, Yamagishi M, et al. Effect of intensive statin therapy on regression of coronary atherosclerosis in patients with acute coronary syndrome: A multicenter randomized trial evaluated by volumetric intravascular ultrasound using pitavastatin versus atorvastatin (JAPAN-ACS [Japan assessment of pitavastatin and atorvastatin in acute coronary syndrome] study). $\mathrm{J} \mathrm{Am}$ Coll Cardiol 2009; 54: 293-302.

34. Motoyama S, Sarai M, Harigaya H, Anno H, Inoue K, Hara T, et al. Computed tomographic angiography characteristics of atherosclerotic plaques subsequently resulting in acute coronary syndrome. J Am Coll Cardiol 2009; 54: 49-57.

35. Budinski D, Arneson V, Hounslow N, Gratsiansky N. Pitavastatin compared with atorvastatin in primary hypercholesterolemia or combined dyslipidemia. Clin Lipidol 2009; 4: 291-302.

36. Sattar N, Preiss D, Murray HM, Welsh P, Buckley BM, de Craen AJ, et al. Statins and risk of incident diabetes: A collaborative meta-analysis of randomised statin trials. Lancet 2010; 375: $735-742$.

37. Huang CH, Huang YY, Hsu BR. Pitavastatin improves glycated hemoglobin in patients with poorly controlled type 2 diabetes. $J$ Diabetes Investig 2016; 7: 769-776.

38. Thakker D, Nair S, Pagada A, Jamdade V, Malik A. Statin use and the risk of developing diabetes: A network meta-analysis. Pharmacoepidemiol Drug Saf 2016; 25: 1131-1149.

39. Barrios V, Escobar C. Clinical benefits of pitavastatin: Focus on patients with diabetes or at risk of developing diabetes. Future Cardiol 2016; 12: 449-466.

\section{Supplementary Files}

Supplementary File 1

Table S1. Percent change in lipid parameters: start of statin treatment to 12-month follow-up

Table S2. Adverse events in the safety analysis set

Please find supplementary file(s);

http://dx.doi.org/10.1253/circj.CJ-17-0419 